\title{
A Rapid Pathogenicity Assay of $R$. solanacearum in 10-12 Days Old Eggplant (Solanum melongena) Seedlings by Root Inoculation Approach
}

\section{Niraj Singh}

Department of Molecular Biology and Biotechnology, Tezpur University, Assam, India, 784028

Department of Microbiology, Royal Global University, Guwahati, Assam, India 781035

\section{Abstract}

This study has reported standardization of pathogenicity assay of Ralstonia solancearum in eggplant seedlings by root inoculation approach. Though the approach is similar to my earlier published work that done with tomato seedlings, in case of eggplant, duration of the seed germination, age of the seedlings, symptom timing and progression rate of the disease are different. In an introductory observation, $R$. solanacearum $\mathrm{F} 1 \mathrm{C} 1$ inoculation was performed in to 10-12 days old eggplant seedlings resulted in lethal infection in eggplant seedlings and subsequently most of these infected seedlings got wilted within 10 days of post-inoculation. Colonization studies of $R$. solanacearum in wilted as well as infected eggplant seedlings were confirmed by GUS staining as well as fluorescence microscopy. In addition, we also observed that wrapping of the eggplant seedlings root by a thin layer of cotton soon after the pathogen inoculation in the root, enhanced the disease progression and wilting of the inoculated seedlings. The standardized root inoculation protocol in eggplant seedlings was found to be efficient to distinguish mutant strains of different virulence genes such as $h r p B$, phcA and pilT from wildtype $R$. solanacearum. Due to its reproducibility and consistency in different eggplant cultivars, the standardized protocol described here is practically observed as an useful and rapid approach to investigate $R$. solanacearum pathogenicity and disease progression at the early seedling stage of eggplant.

\section{Introduction}

R. solanacearum is a soil-born bacterium, the causal agent of bacterial wilt disease in many plant species including eggplant and other vegetable crops (Wicker et al., 2007; Genin, 2010). $R$. solanacearum infection and wilting is not only localized to its specific host, but also it 
spreads from one host to the other through soil, contaminated water and weeds (Genin, 2010). In spite of broad host range of this pathogen, only few host plants such as tomato, potato and Arabidopsis thaliana have been used mainly for studying pathogenicity. Similar to the tomato plants, bacterial wilt is also a very common disease in eggplants in tropical as well as in subtropical regions (Gaitonde and Ramesh; Smith, 1896; Shekhawat et al., 1978; Ramesh et al., 2009; Salgon et al., 2017). Eggplants one of the most common vegetables, consumed in various ways by a large population across the world and it is a rich source of protective nutrients and fibers (Hedges and Lister, 2007). Eggplant belongs to solanaceae family, it is an agriculturally important crop plant for pathogenicity and bacterial wilt related study. Till now, determination of $R$. solanacearum pathogenicity factors in case of eggplant has not been addressed appropriately in comparison to tomato or some other solanaceae crop. Studies on $R$. solanacearum pathogenicity in eggplants have mainly been to screen resistant cultivars against the bacterial wilt disease and to investigate resistant mechanisms of eggplant against the wilt pathogen (Artal et al., 2013; Gopalakrishnan et al., 2014).

In addition to that, most of solanaceae family crop plant species such as tomato, potato, and pepper etc. have originated from the new world, while eggplant and its associated wild relatives belongs to the old world. It is being assumed that eggplant was domesticated in Southeast Asia region (Daunay, 2008; Syfert et al., 2016). While on the genetic level, both tomato and eggplant are autogamous diploid plant species with twelve chromosomes and nearly equal genome size (Arumuganathan and Earle, 1991), therefore, deep investigation and study on co-evolution between eggplant and its associated old world pathogen relationship may point out why some specific resistance genes do not exist in the new world solanaceous crops plant (Hirakawa et al., 2014; Nahar et al., 2014). Along with its agronomical importance, eggplant genomics are also less studied and documented in comparison to other major solanaceous crops like tomato and potato. A study was also carried out in eggplant under controlled environment against a core collection of twelve strains, representative of all the exiting phylotypes of $R$. solanacearum (Lebeau et al., 2011). The result of that study indicated that eggplant is a potential host for most of the existing phylotypes of $R$. solanacearum. Thus, pathogenicity study in eggplant as well as comparative virulence and disease progression study between other common hosts like tomato, eggplant might be helpful for in-depth understanding on molecular mechanism of plant-microbe interaction during infection and wilting. This study can also suitably project 
eggplant seedling as a suitable model for mapping and investigating resistance factors to pathogens as well as other determinates. On the basis of severity of wilt disease and economic importance of this crop, the present investigation of $R$. solanacearum pathogenicity behavior and disease progression study in eggplant seedlings will be very helpful to generate necessary information for the understanding of eggplant and $R$. solanacearum interaction during infection and wilting. Over and above, this study will be also very significant for the economic as well as scientific interest for suitable management measures to minimize the substantial crop loss.

\section{Materials and Methods}

\subsection{Bacterial strains, growth media and culture conditions}

$R$. solanacearum as well as other bacterial strains recruited in this work were the same strains which have been listed in the recently published tomato seedlings work (Singh et al., 2018). Growth medium and antibiotics were also used as same as mentioned in the tomato seedlings work. R. solanacearum F1C1 (Kumar et al., 2013) and its derivative mutant strains as well as Pseudomonas putida were grown in BG medium (Bacto glucose agar medium) (Boucher et al., 1985) supplemented with glucose $(0.5 \%$, w/v). Incubation temperature for $R$. solanacearum strains and Pseudomonas putida was $28^{\circ} \mathrm{C}$ while Bacillus subtilis as well as Escherichia coli strains were grown in LB medium (Bertani, 1952) at $37^{\circ} \mathrm{C}$ in the same way as discussed in case of tomato seedlings . Agar $(1.5 \%, \mathrm{w} / \mathrm{v})$ was added in case of solid medium if and when necessary.

\subsection{Germination of eggplant seedlings for inoculation}

Seeds of different eggplant cultivars employed during pathogenicity assay standardization were pre-soaked in sterile distilled water for 5-6 days. Further, this was followed by spreading of the soaked seeds on sterile and wet tissue paper bed in a plastic tray. After that, plastic tray was covered with transparent polyethylene sheet and allowed for germination in a growth chamber (Orbitek, Scigenics, India) maintained at $28^{\circ} \mathrm{C}, 75 \%$ relative humidity $(\mathrm{RH})$ and $12 \mathrm{~h}$ photoperiod respectively. It usually took 2-3 days for sprouting of seeds. Just after sprouting, polyethylene sheet cover was removed from the tray, sterile distilled water was sprinkled at regular time interval till 7-8 days to sustain the germination process and growth of seedling in a better way. Here the age of the eggplants seedlings have been defined from the day of spreading the soaked seeds for germination on the wet tissue paper bed in the plastic tray. 


\subsection{Preparation of bacterial inoculums}

$R$. solanacearum $\mathrm{F} 1 \mathrm{C} 1$ as well as other bacterial strain inoculums were prepared by following exactly the same procedure used in case of tomato seedlings inoculation as mentioned in case of tomato seedling(Singh et al., 2018) .

\subsection{Root inoculation of $R$. solanacearum in eggplant seedlings}

In case of eggplant, around 15-20 ml of $R$. solanacearum $\mathrm{F} 1 \mathrm{C} 1$ inoculum $\left(\sim 10^{9} \mathrm{CFUml}^{-}\right.$ ${ }^{1}$ ) was taken in a sterile container similar to tomato seedling inoculation as mentioned in case of tomato (Singh et al., 2018). After that, from the germinated seedling bed, 10-12days old eggplant seedlings were picked one at a time. Root of each seedling was then carefully dipped (up to the root-shoot junction) into the bacterial inoculum for 1-2 seconds followed by transfer of the seedling to an empty and sterile microfuge tube of 1.5 or $2.0 \mathrm{ml}$ (Figure: 1A \& 1B). All the eggplant seedlings were inoculated by the same procedure as mentioned in earlier infection assay in tomato seedlings.

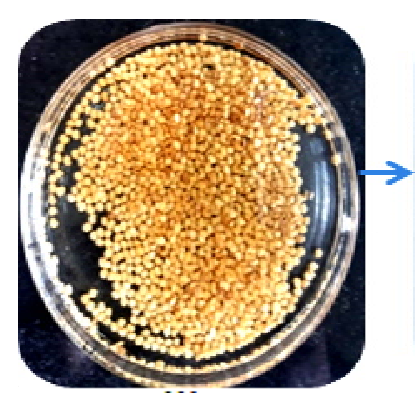

(A)

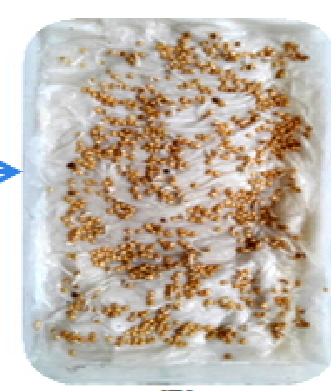

(B)

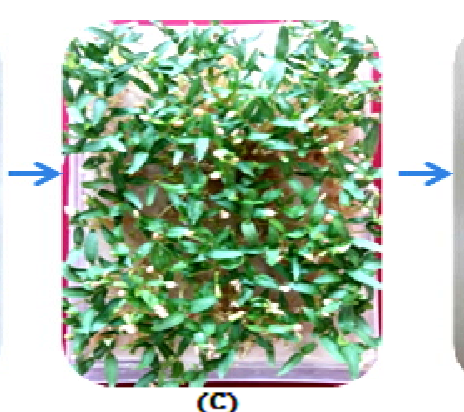

(C)

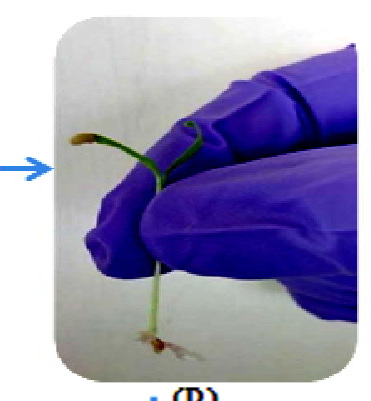

$V^{(D)}$
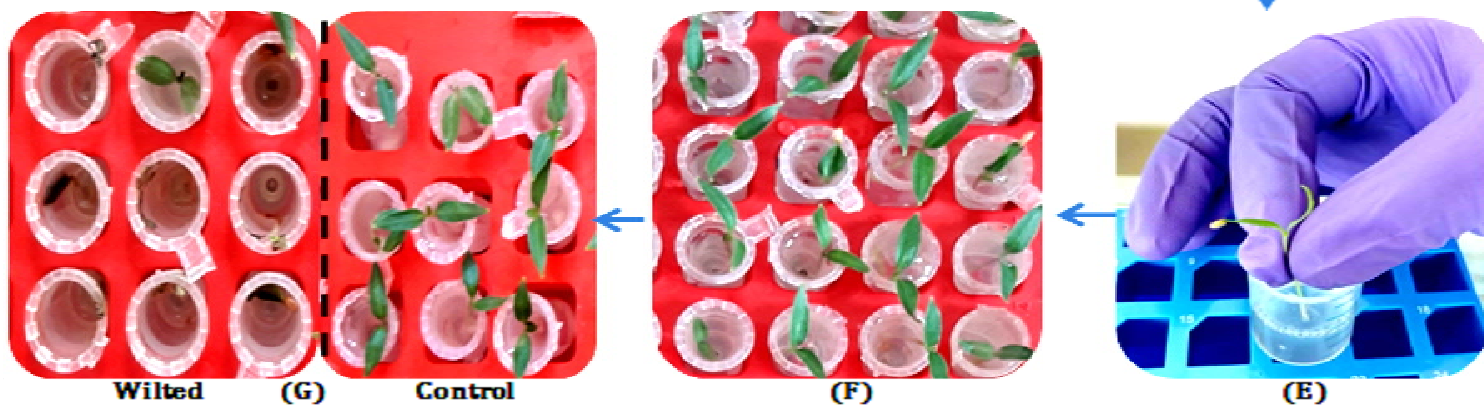

(E)

Figure 1A: Pictorial presentation of root inoculation method in eggplant seedlings

(A) Soaking of eggplants seeds in sterile distilled water, (B) Germination of eggplant seeds on cotton bed, (C) Germinated seedlings in plate after 10-12 days, (D) A 10-12 days old eggplant seedling, (E) Root inoculation of eggplant seedling in pathogen 
inoculum, (F) Transfer of each inoculated seedling to sterile empty microfuge (1.5-2.0 $\mathrm{ml}$ ) tubes and after five minutes exposure to air, sterile water is added in the microfuge, (G) After 10 days, 80\%-90\% of infected tomato seedlings were wilted/died (left side) in comparison to water as control (right side).

In all these experiments, a set of forty eggplant seedlings was considered for each pathogenicity test per inoculum used. In control set also, 40 (forty) seedlings were mock-inoculated with sterile distilled water. All the inoculated eggplant seedlings along with control were transferred to a growth chamber maintained at $28^{\circ} \mathrm{C}, 75 \% \mathrm{RH}$, photoperiod of $12 \mathrm{~h}$. The inoculated seedlings were analyzed for disease progression and wilting from next day onwards (inoculation) till $10^{\text {th }}$ day of post-inoculation and findings were recorded accordingly.

During this work, seedlings of four different commercially available eggplants cultivars such as Akshay (Bharat nursery Pvt. Ltd.), Lalita (Bharat nursery Pvt. Ltd), Debgiri (Debgiri Agro Products Pvt. Ltd.) and 749 (Pahuja Seed Pvt. Ltd.) were tested for $R$. solanacearum pathogenicity and disease progression by the root inoculation method as described above.

Root inoculation of $h r p B$, phcA, pilT and $r p o N_{2}$ mutant strains of $R$. solanacearum as well as non-pathogenic bacterial strains such as $B$. subtilis, $P$.

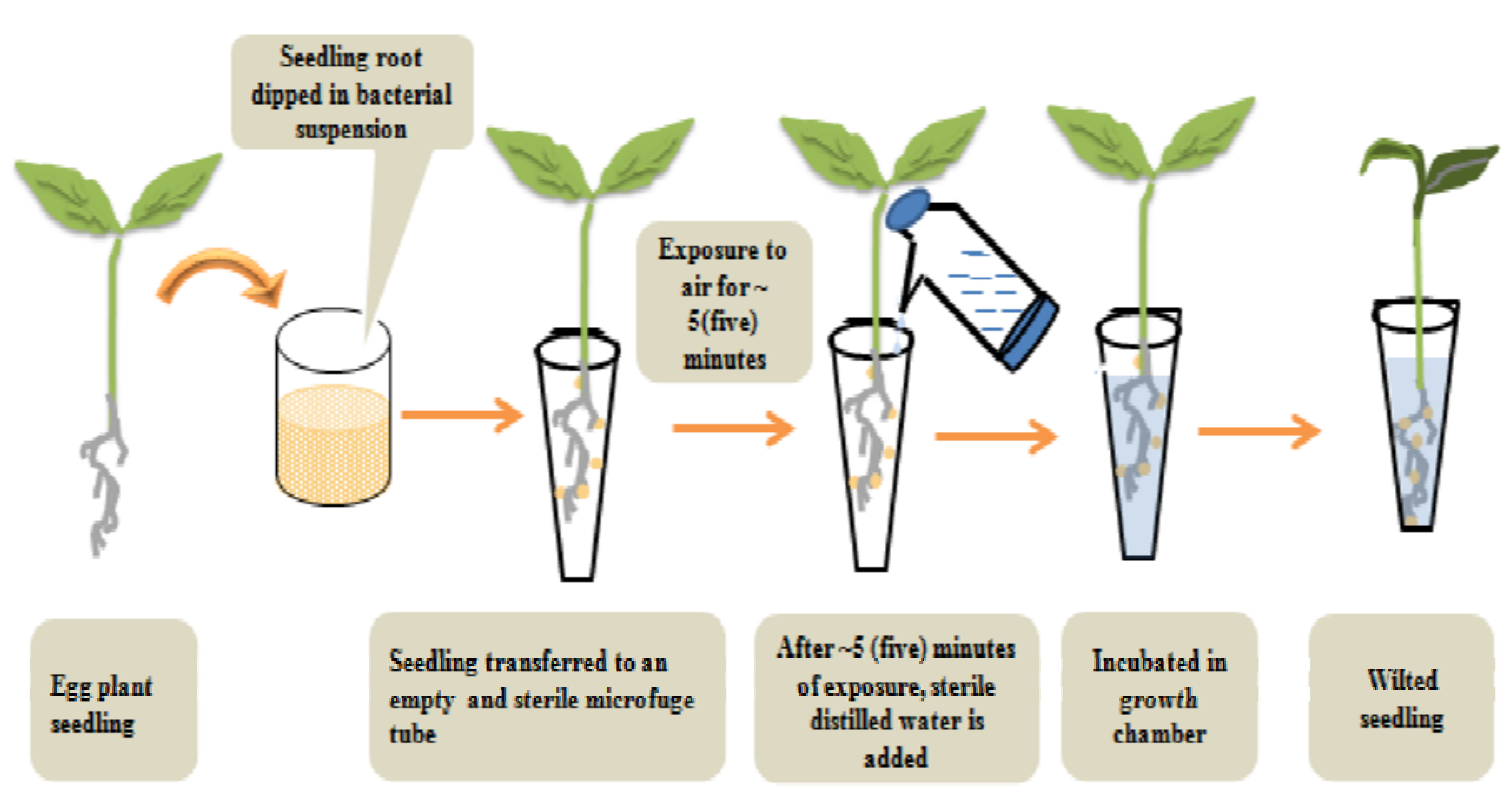

Figure 1B: Schematic representation of the crucial steps of root inoculation method in eggplant seedlings 
A schematic representation emphasizing the exposure to air after pathogen inoculation and various steps during the pathogenicity assay of $R$. solanacearum in 10-12 days old eggplant seedlings by root inoculation method

putida, and E. coli in eggplant seedlings were carried out in the same way as mentioned in case of tomato seedlings (Singh et al., 2018).

\subsection{Colonization study of $\boldsymbol{R}$. solanacearum in eggplant seedlings}

Grown culture of TRS1002 (GUS marked), TRS1016 (mCherry marked)(Monteiro et al., 2012; Capela et al., 2017) as well as TRS1017 (GFP marked) strain of $R$. solanacearum were pelleted down by centrifugation and $10^{9} \mathrm{CFU} / \mathrm{ml}$ inoculum was prepared by the same procedure described for $R$. solanacearum in tomato seedlings work (Singh et al., 2018). Thereafter, bacterial inoculums were used for infection by root inoculation approach in 10-12 days old eggplant seedlings in the same way as stated above. After third day of post inoculation, eggplant seedlings were employed for colonization study followed by the same procedure as mentioned in the case of tomato seedlings.

\subsection{Study of cotton fiber wrapping on the inoculated root of eggplant seedling and its impact on wilting}

During the standardization process of $R$. solanacearum pathogenicity assay in early seedling stage of eggplant, this study has gone through many trial and error among the different steps of pathogenicity standardization process. During 


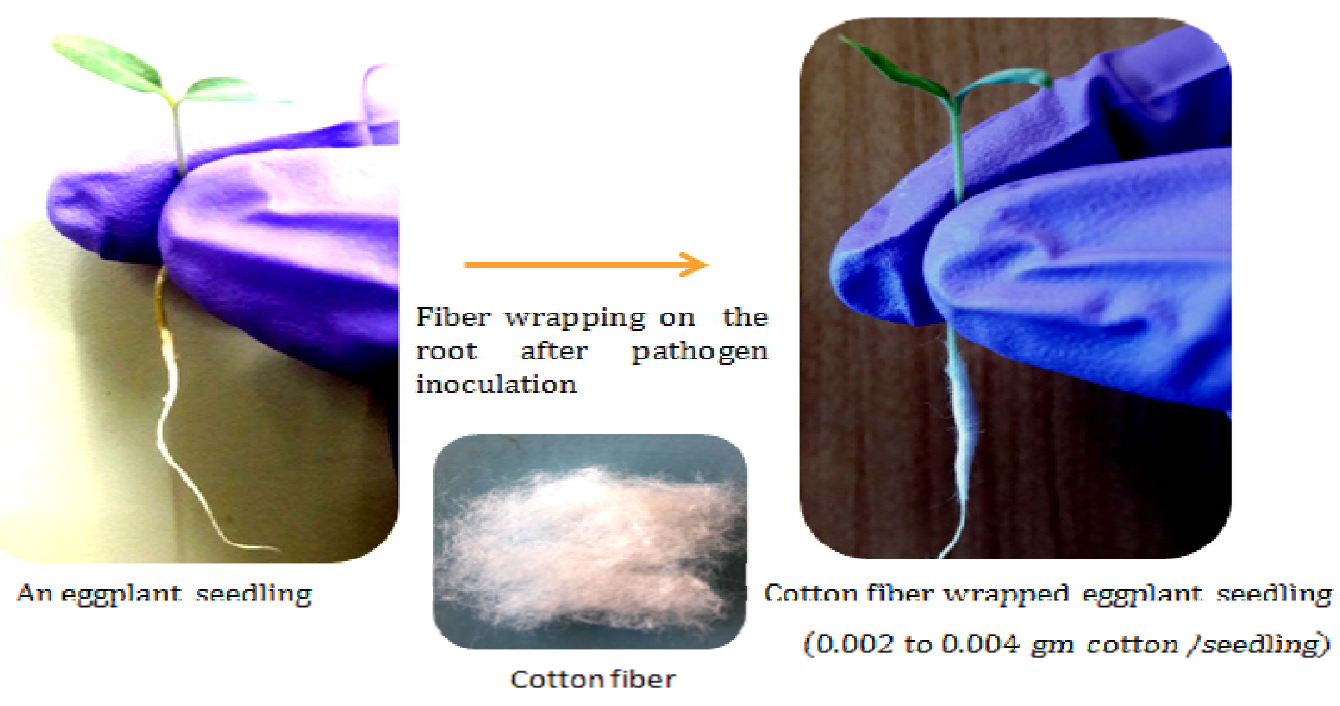

Figure 2: Pictorial presentation of the steps of cotton wrapping on the root of eggplant seedlings

This figure is showing the 10-12 days old eggplant seedling before and after cotton fiber wrapping process on root part of eggplant seedling just after the pathogen inoculation process.

standardization it was observed that a thin layer of cotton fiber if wrapped on the root of eggplant seedling just after the pathogen inoculation, it used to enhance the wilting and disease progression as compared to those set of seedlings without the wrapping of cotton fiber (Figure 2). During, pathogen inoculation process, we have performed in the same way as motioned above, after that a thin layer of sterile cotton fiber was wrapped around the seedling root up to root shoot junction (Figure 3). After that, infected seedlings were transferred to sterile microfuge tube and allowed for $\sim 5$ (five) minutes of air exposure. Thereafter, water is added to the microfuge in the same way as described in the above sections as well as in the previous chapter. 


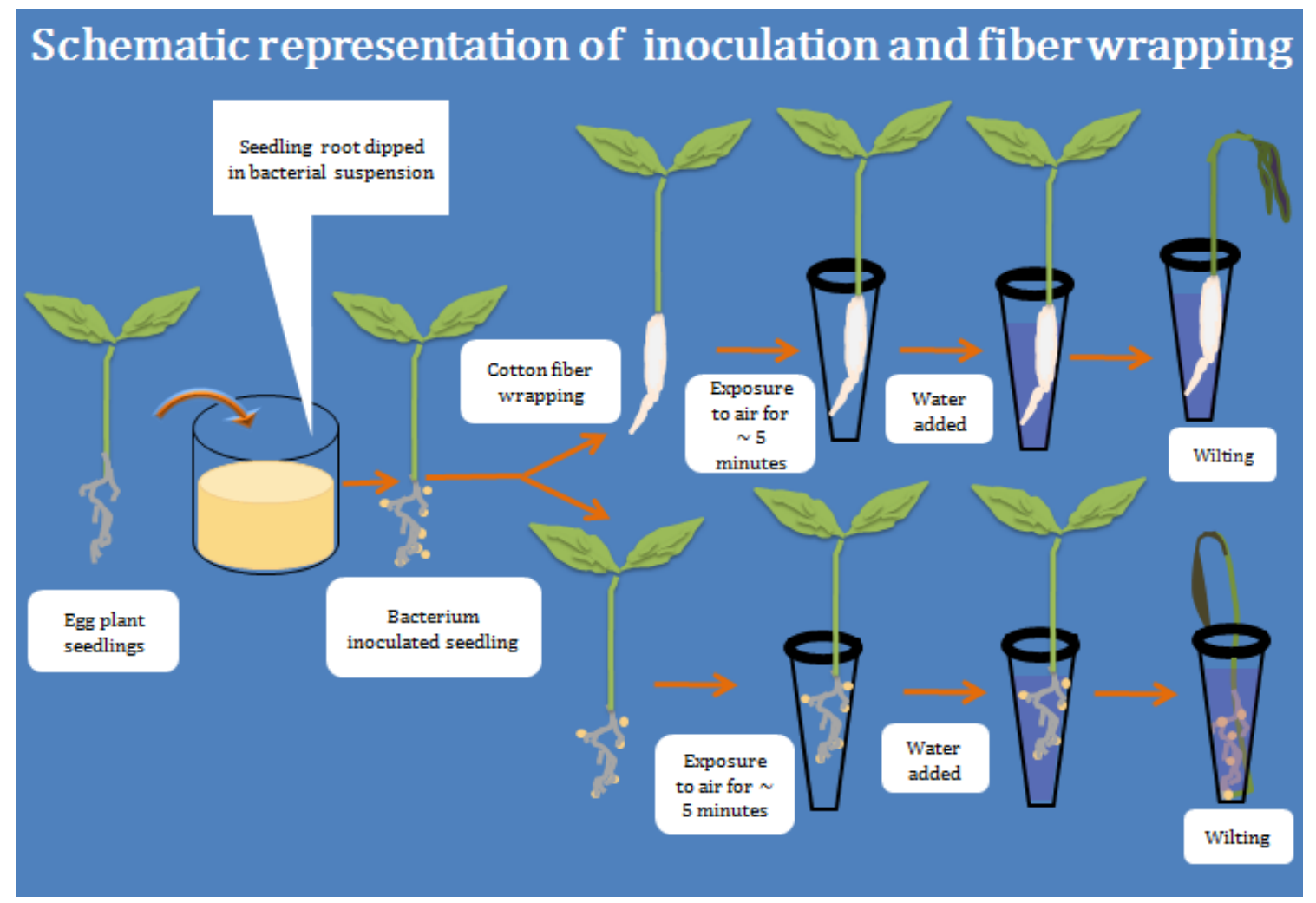

Figure 3: Schematic representation of pathogen inoculation and cotton fiber wrapping on the root of eggplant seedlings

A self-explanatory sketch describing how sterile cotton was wrapped around the root of eggplant seedling just after pathogen inoculation step. The whole process was used to understand the impact of cotton fiber on $R$. solanacearum wilting in the eggplant seedlings by comparing pathogenicity between cotton wrapped and unwrapped set of infected eggplant seedlings.

\section{Results}

\subsection{R. solanacearum pathogenicity in eggplant seedlings by a root inoculation method}

In the earlier published work, development of pathogenicity assay and wilt disease progression in 6-7 days old tomato seedlings were studied and susceptibility of early stages of tomato seedlings toward $R$. solanacearum wilting was also validated. In addition to that, this 
early seedling stage pathogenicity study approach was found to be very much consistent and proficient. In wake of testing reproducibility of this newly standardized pathogenicity approach in eggplant seedling as another most affected host, we have carried out this work. In the inoculation process, we have followed the same steps as mentioned in tomato seedlings. For pathogenicity standardization, 10-12days old eggplant seedlings were inoculated with $R$. solanacearum suspension, thereafter inoculated seedlings sets along with controls were transferred to growth chamber for the observation of disease progression. On the $10^{\text {th }}$ day postinoculation, about $80-90 \%$ of the inoculated eggplant seedlings were found to be dead due to disease and wilting (Figure 4).

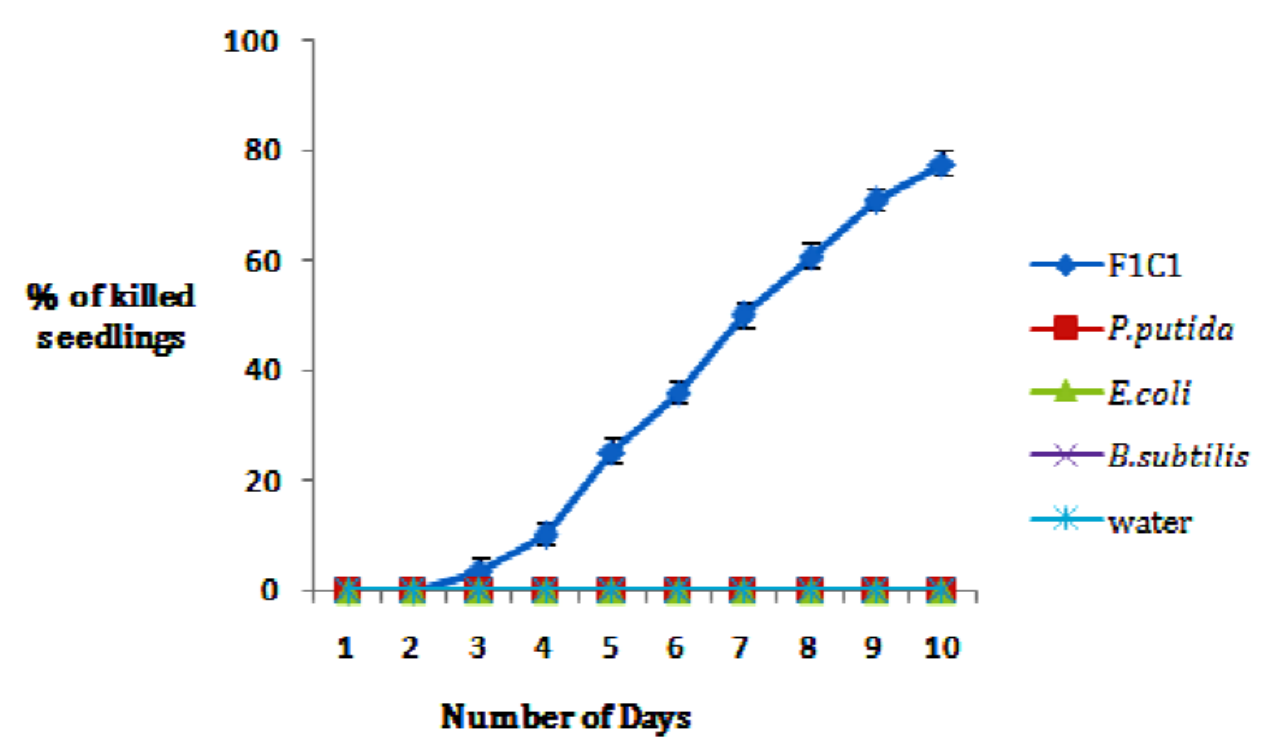

Figure 4: Pathogenicity assay of F1C1 and other bacterial strains in eggplant seedlings

This graph is showing the wilting or death occurred only in F1C1 infected eggplant seedlings. In case of $P$. putida, B. subtilis and $E$. coli, no killing was observed which was similar to water as control.

For further confirmation of the wilting of inoculated eggplant seedlings by root inoculation approach specific to $R$. solanacearum, eggplant seedlings were also inoculated with some well-known non-pathogenic bacteria such as $P$. putida, B. subtilis, and E. coli along with sets of $R$. solanacearum infection (Figure 4). After $10^{\text {th }}$ day of post-inoculation, it is observed that none of the eggplant seedlings exhibited disease symptom inoculated with these nonpathogenic bacteria. This result of infection clearly indicated that wilting and death of the eggplant seedlings 
occurred only due to inoculation with $R$. solanacearum F1C1. Further, to confirm reproducibility of this standardized root inoculation approach, it was again tested on three different eggplant cultivars such as Lalita, Debgiri and 749. Magnitude of $R$. solanacearum F1C1 pathogenicity could be observed as similar kind in all three eggplant cultivars (Figure 5 \& 6).

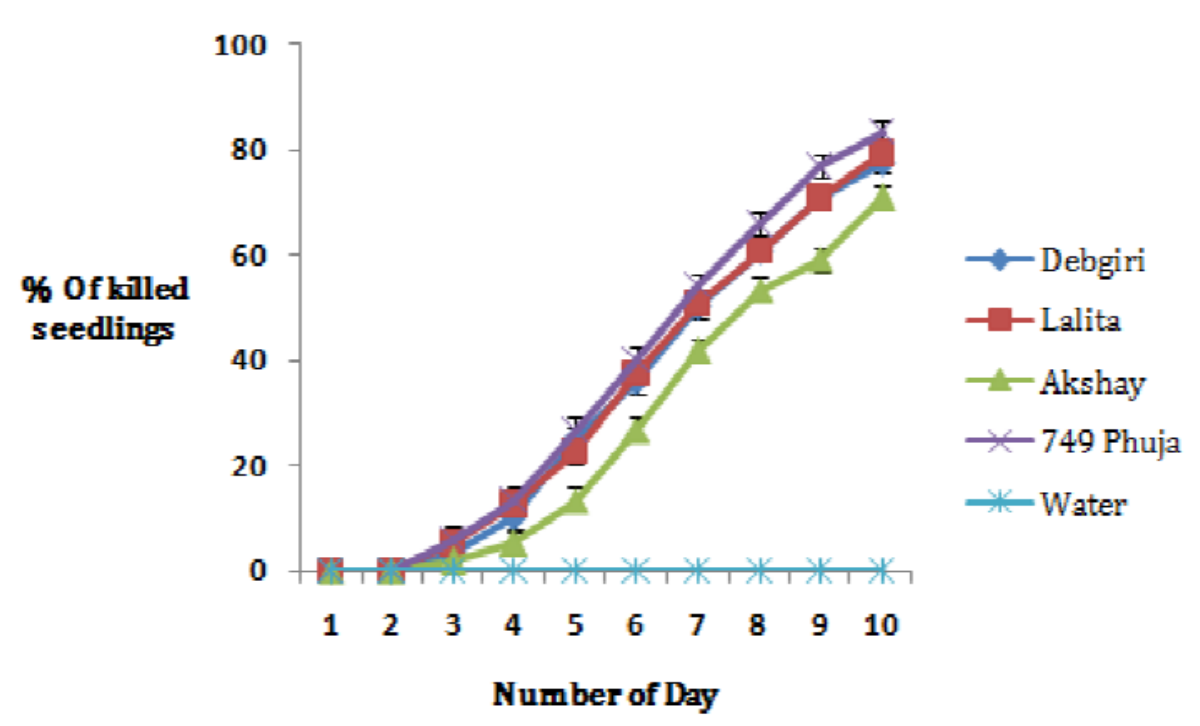

Figure 5: Pathogenicity assay in different eggplant cultivars

This graphis showing the pathogenicity assay of $R$. solanacearumF1C1 in four different eggplant cultivars by root inoculation method. It can be clearly observed that this $R$. solanacearum F1C1 pathogenicity by this inoculation method was nearly equal to virulence and disease progression in all the four different eggplant cultivars.

\subsection{Colonization study}

Colonization study in eggplant seedling was carried out by using $R$. solanacearum F1C1 derived strains such as TRS1002 (GUS marked), TRS1016 (mCherry marked) as well as TRS1017 (GFP marked). Bacterial colonization in the infected seedlings was observed from root to the shoot regions, which were 


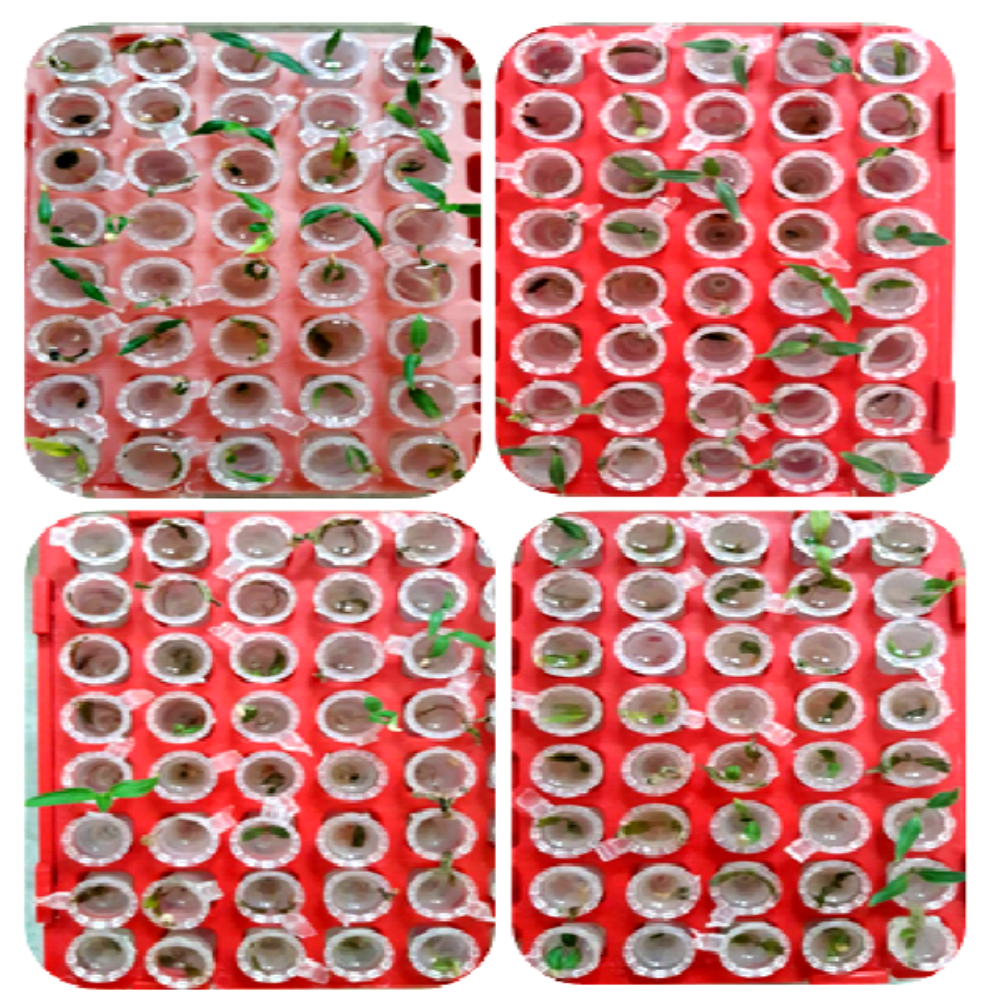

Figure 3.6: Pictorial presentation of pathogenicity assay in four different eggplant cultivars by root inoculation

These pictures are showing the pathogenicity assay of $R$. solanacearum F1C1 in four different eggplant cultivars by root inoculation method. It can be clearly observed that this $R$. solanacearum F1C1 pathogenicity by root inoculation method was highly reproducible in all the four different eggplant cultivars. in left side upper and lower picture indicating Akshay and 749 Pahuja cultivar while in right side upper and lower picture indicating Debgiri and Lalita. Wilting and disease progression was found to be consistent in respective cultivar.

confirmed by GUS staining as well as by red fluorescence (Figure .7). This result of colonization investigation suggested the growth and colonization of the pathogen from root to the shoot regions during the infection process and disease progression.

\subsection{The root inoculation method can be used to study $R$. solanacearum and its mutant derivatives virulence functions}

To further validate and find out the usefulness of this standardized root inoculation protocol to study the pathogenicity functions of $R$. solanacearum eggplant seedlings, seedlings 
root were inoculated with different $R$. solanacearum mutant strains such as hrpB (TRS1012), phcA (TRS1013), pilT (TRS1014) and rpoN2 (TRS1015) in the similar way as mentioned in the previous

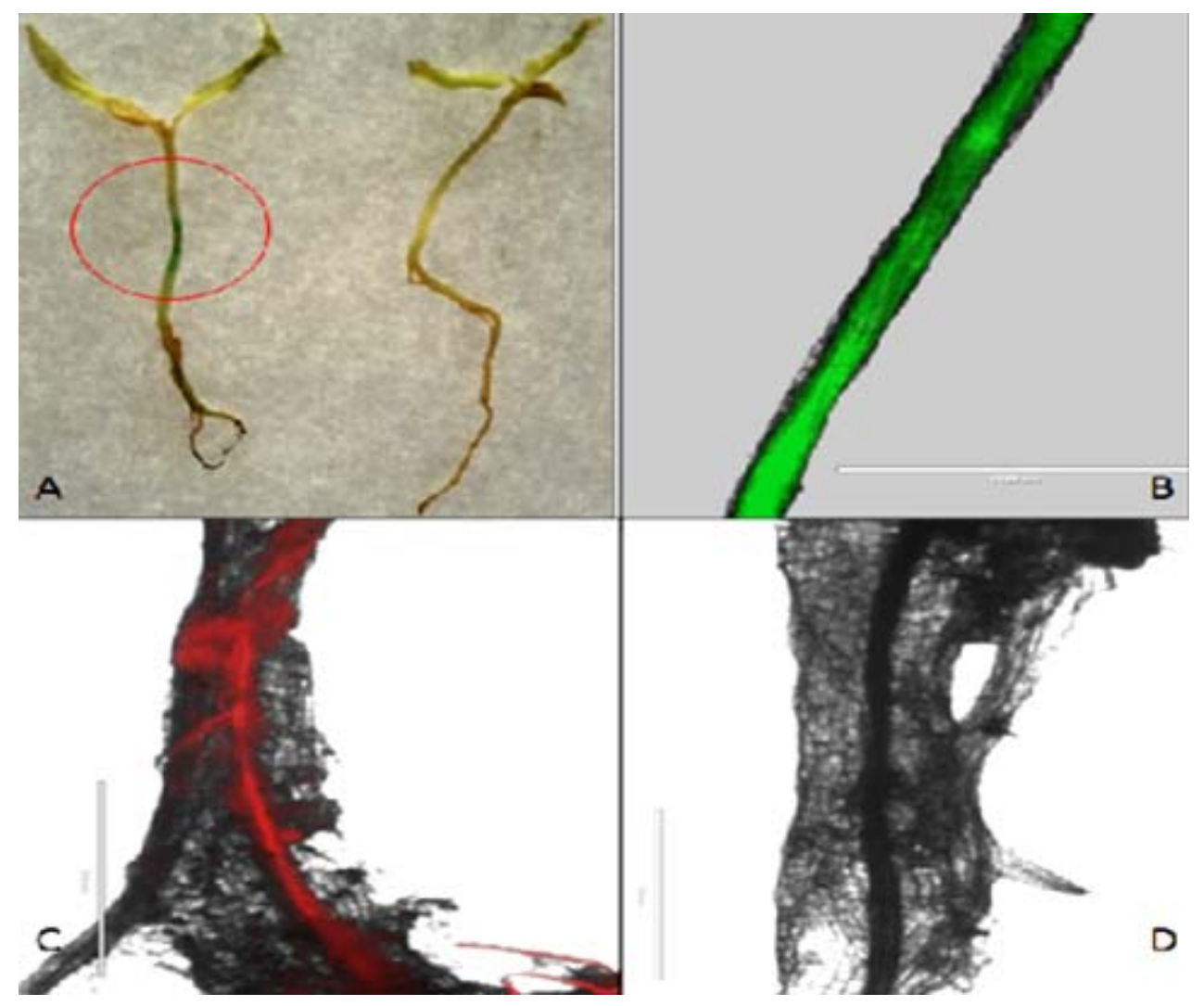

Figure 7: Pathogen colonization study with the help of GUS staining and fluorescence microscopy in eggplant seedlings

(A) is showing GUS staining (on the stem) for colonization confirmation (red circle) while right side seedling iscontrol showing no blue color, (B) is showing green fluorescence in root region which is confirming the colonization of GFP tag $R$. solanacearum strain, (C) is showing the red fluorescence in root region which is confirming the presence and colonization of m-cherry tagged $R$. solanacearum inside tomato seedling while (d) is showing the root region of control seedling with the absence of any florescence.

Seedling published work. As assumed, the $h r p B$ mutant strain was found to be non-pathogenic and the $p h c A$ mutant strain was observed to be highly reduced for virulence. The pilT mutant was reduced for virulence but more virulent than the $p h c A$ mutant. In case of rpoN2 mutant strain, the 
virulence proficiency was found to be similar to the wild type $R$. solanacearum F1C1 (Figure 8). The virulence phenotypes and disease progression of $h r p B$, phcA, pilT and rpoN2 mutants were in concordance with the virulence phenotype data of tomato seedling (mentioned in previous chapter) as well as in grownup plant as reported in literature (Ray et al., 2015) .

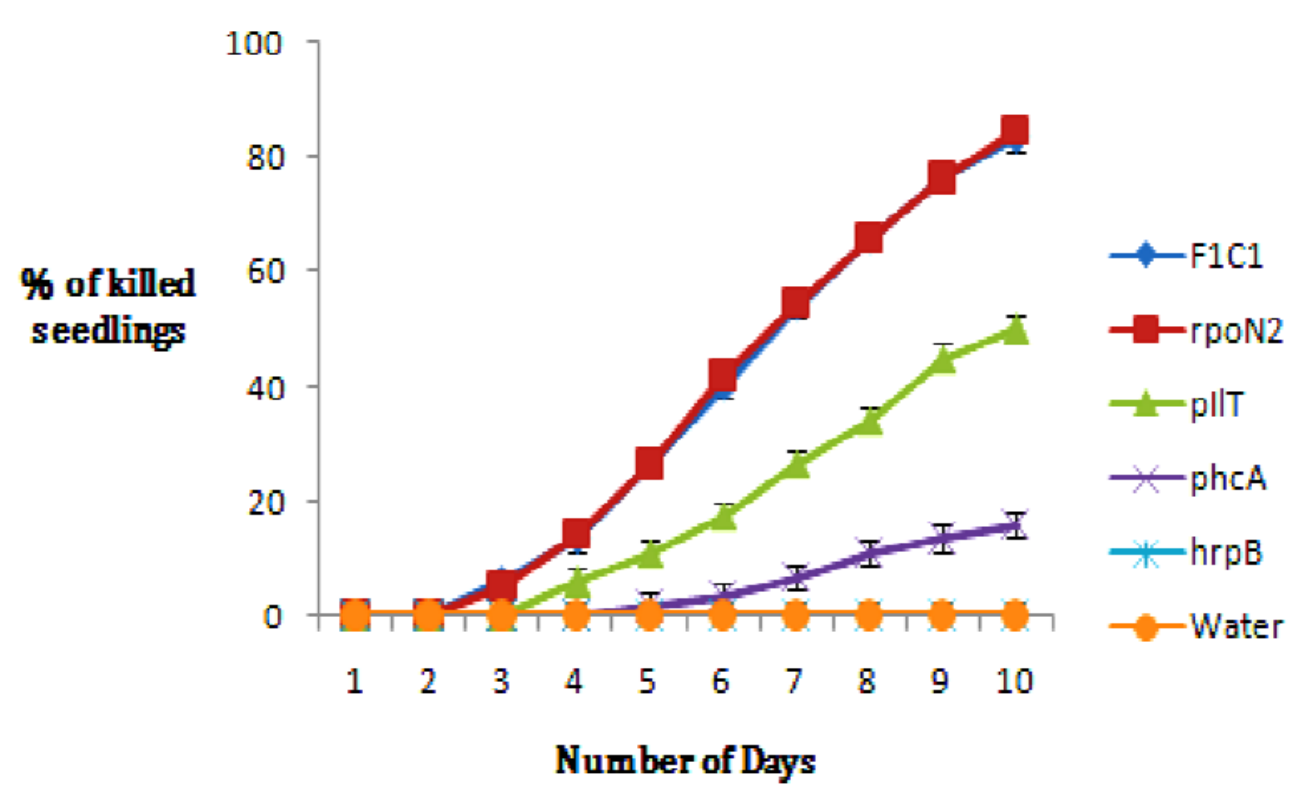

Figure 8: Comparative pathogenicity assay of F1C1 and other derivative mutant strains in eggplant seedlings.

This graph is showing the pathogenicity comparison of $\mathrm{F} 1 \mathrm{C} 1$ to its derivative mutantsrpoN2, hrpB, phcA and pilT. The number (\%) of killed seedlings in case of phcA and pilT were much lesser than the wild type (F1C1). While in case of $h r p B$, no wilting was observed similar to water control set. But in case of rpoN2, there was no affect of pathogenicity similar to wild type F1C1.

\subsection{Wrapping of thin layer cotton fiber around eggplants seedling root to study its impact}

on $\boldsymbol{R}$. solanacearum pathogenicity

During the standardization of pathogenicity assay in eggplant seedlings, it was observed that if a thin layer of cotton fiber was wrapped around the root of eggplant seedlings just after the pathogen inoculation, wilting and disease progression was found to be enhanced in comparison to infected eggplant seedling (sets) without cotton wrapping (Figure $9 \& 10$ ). 
The effect of cotton wrapping around the root of eggplant seedling was an interesting observation of this study. But to understand and reveal the exact reason behind the enhancement of virulence and pathogenicity, there is a necessary of further dipper investigation of plantmicrobe interaction behavior during early stage of infection and attachment.
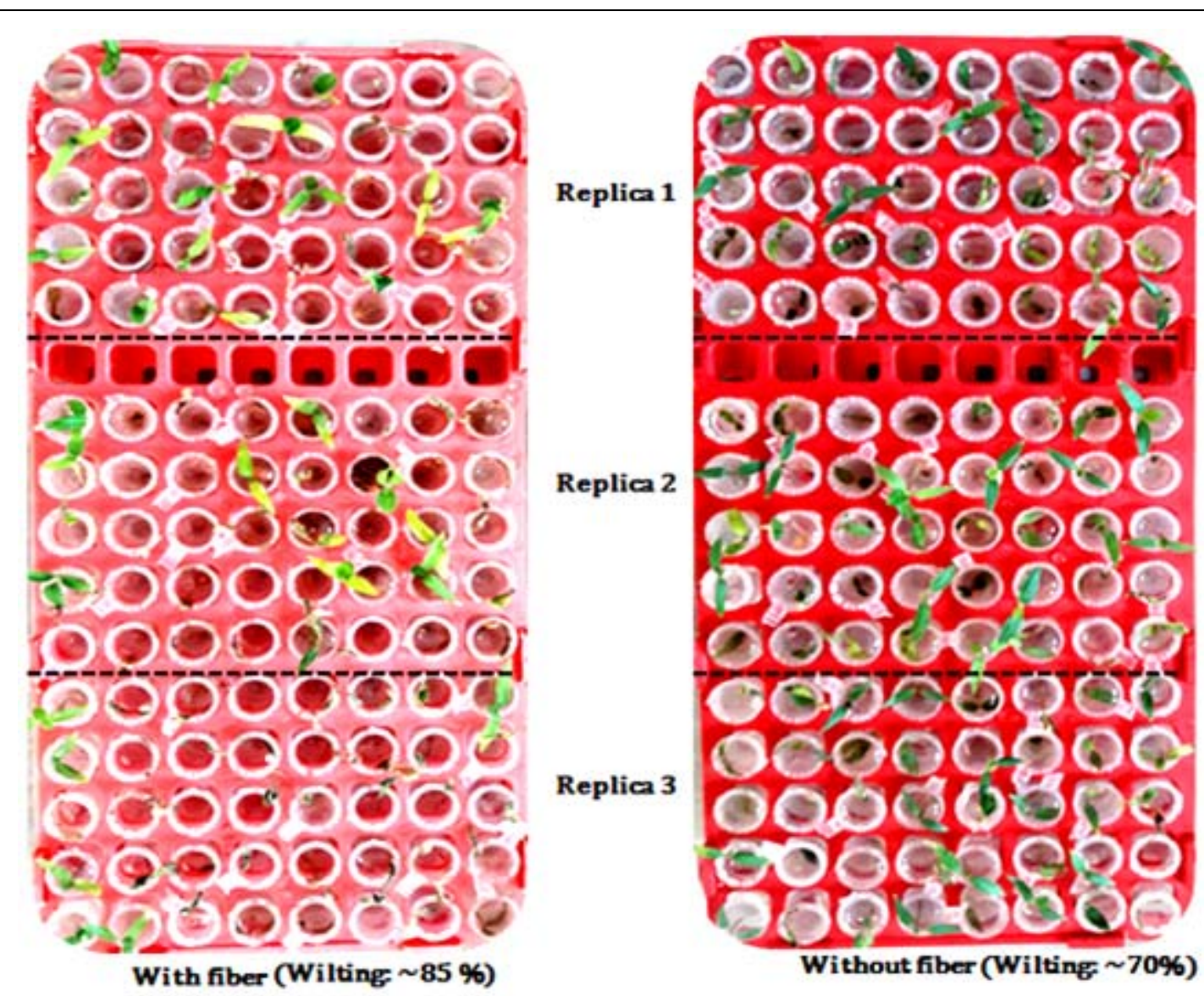

Figure 9: Comparative pathogenicity assay between wrapped and unwrapped eggplant seedlings

The picture in the left side(cotton wrapped)with a set of seedling are exhibiting more number of wilting in comparison to set of infected seedlings in the right side (unwrapped) picture. In addition to that, seedling leaves in the left sidepicture are yellowish in comparison to rightside which is also supporting pathogenicity enhancement in cotton wrapped seedlings.

\section{Discussion}

In this work, we are documenting the standardization of a protocol to study $R$. solanacearum pathogenicity (by root inoculation approach) in 10-12 days old eggplant seedlings 
under gnotobiotic and additional nutrients free conditions. This standardized protocol was found to be very efficient and consistent for the pathogenicity assay at seedlings stage of eggplant. In addition to that, it has also shown the reproducibility in other different eggplants cultivars. This newly standardized protocol is successfully validated with the help of known mutant gene function of $R$. solanacearum derivatives such as $h r p B$, phcA and pilT mutant strains. Over and above, this inoculation process is also found to be easy to investigate the pathogen colonization study at seedling

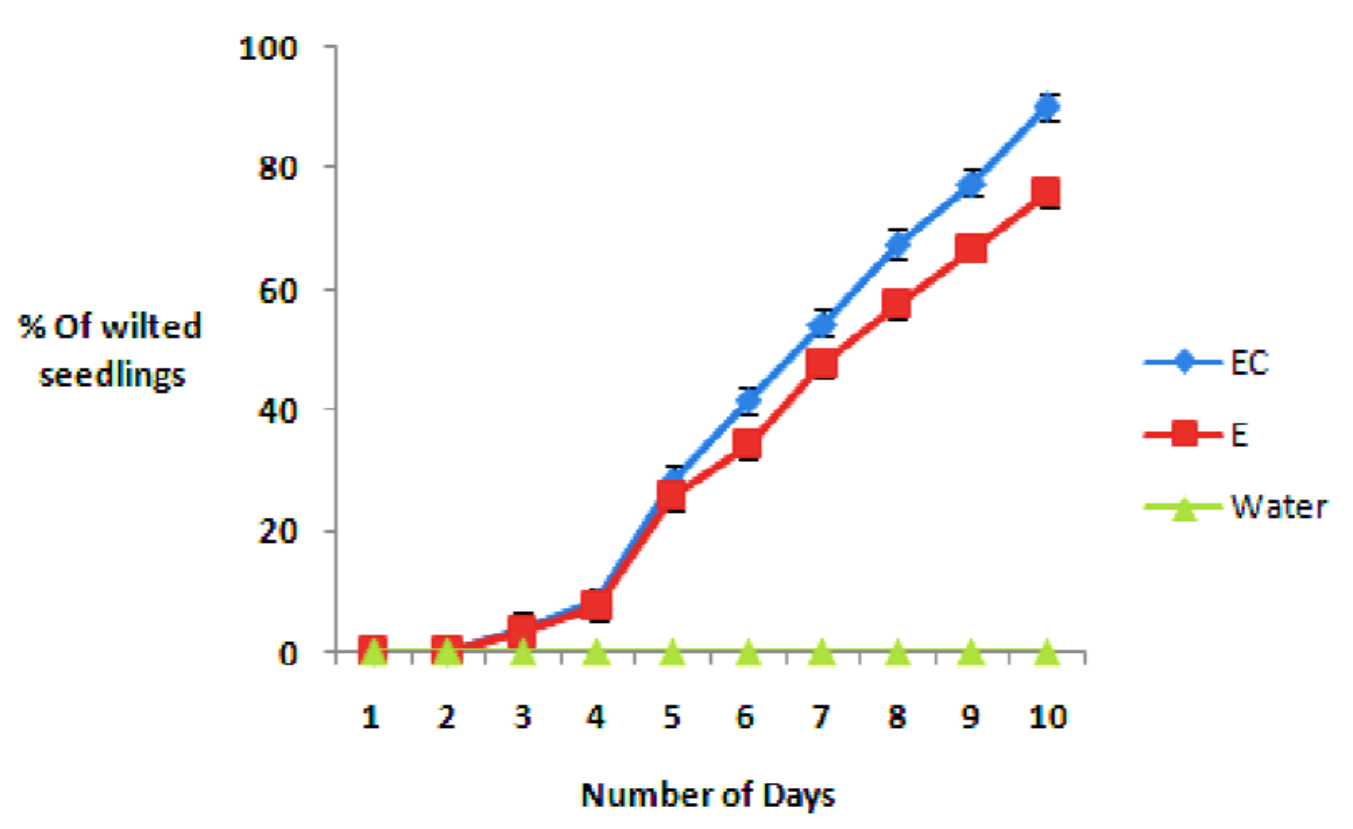

Figure 10: Pathogenicity assay in cotton wrapped and unwrapped eggplant seedlings(statistical analysis : Appendix II)

This picture is showing the pathogenicity assay of F1C1 in 2 (two) different sets of an eggplant cultivar by root inoculation method. In this graph, EC is indicating eggplant with the wrapping cotton fiber while $\mathrm{E}$ is indicating the eggplant without the wrapping of cotton fiber. This graph is clearly showing that through this method of inoculation, F1C1 is significantly more virulent and disease progression ( $5^{\text {th }}$ day onwards) in case of EC or cotton fiber wrapped eggplant seedlings.

stage. Colonization study of this bacterial pathogen after post inoculation clearly manifested that the pathogen is successfully associated with the root and shoot regions of the infected seedlings. 
However, this pathogenicity standardization protocol in eggplant seedlings is superficially looks similar to tomato seedling, but actually it has many minute differences. In case of eggplants seedlings, pathogenicity symptom appearance, seed germination process and seedling age were very different from tomato seedlings. It took lot of time and effort to optimize optimum germination of seed as well as disease progression and wilting in eggplant seedlings. In this protocol, seed soaking time in the presence of distilled water for eggplant seeds was optimized as 5-6 days while in case of tomato, 2 days of soaking duration was found to be sufficient to get optimum germination. The exact reason behind the requirement of longer soaking duration for optimum germination about eggplant seeds is yet to know. During standardization, we felt that eggplant seed coat was harder and thicker than that of tomato, which might be a probable reason behind requirement of longer soaking duration of eggplant seeds for optimum germination. The other point was that the initial disease symptom appearance in case of eggplant seedling was little bit late in compression to tomato seedling and the assay got completed by 10 days in eggplant compared to 7-8 days in case of tomato seedling. Over and above it is also important to mention seedling age of eggplant at the time of pathogen inoculation, which was different than tomato: 10-12 days old eggplant seedlings were used for pathogen inoculation and disease progression study as compared to 6-7 days old seedlings of tomato. Therefore, because of several differences of this standardized protocol from the earlier reported protocol for tomato seedlings, we are documenting this study as a separate protocol for pathogenicity assay in eggplant seedling. This standardized protocol for pathogenicity assay is also found to be very much consistent and reproducible in other three different eggplant cultivars. Hence, on the basis of reproducibility and consistency of this protocol, this study is suggesting the carried out protocol may be an efficient way to study plant-microbe and wilt disease progression of $R$. solanacearum at the early seedling stage of eggplant. In addition to that, the pathogenicity assay can be performed by maintaining near to natural mode of inoculation in less resource dependent manner. It is pertinent to note that this is the first standardization report on $R$. solanacearum pathogenicity study in cotyledon stage seedlings of eggplant by root inoculation.

Very interestingly, in-between hit and trial of pathogenicity assay standardization, this study have noticed that wrapping of a thin layer of sterile cotton fiber around the root of the pathogen inoculated eggplant seedling used to enhance the wilting and disease progression of $R$. 
solanacearum. In an initial study we also observed that poly ethylene fiber was enhancing the $R$. solanacearum pathogenicity in eggplant seedlings like that of the cotton fiber. This observation might be important to know the impact fiber structures present in fields for the natural infection R. solanacearum in eggplant cultivars and other hosts. Although exact mechanism is still unknown, probably this cotton fiber used induced some adhesion functions such as type IV pili during initial attachment and/or induced biofilm formation pathway to stimulate additional virulence and wilting in the eggplant seedling (Liu et al., 2001; Siri et al., 2014; Persat et al., 2015).We have not come across any references regarding the impact of fibers on $R$. solanacearum pathogenicity in host plants. In future, it will be interesting to investigate and understand the exact mechanism and correlation of cotton fiber in disease progression and wilting.

\section{Acknowledgment}

Niraj Singh is thankful to Tezpur University, Assam, India as well as Royal Global University, Assam, India for providing an opportunity to work. Over and above, Niraj Singh is also very much thankful to Prof. Suvendra Kumar Ray, Tezpur University, for motivation and kind support. In addition to that, Niraj Singh also thankful to Department of Biotechnology, Government of India for the DBT JRF and SRF fellowship and providing necessary facilities.

\section{References:}

- Artal RB, Gopalakrishnan C, Thippeswamy B. An efficient inoculation method to screen tomato, brinjal and chilli entries for bacterial wilt resistance. Pest Management in Horticultural Ecosystems 2013;18(1):70-73.

- Arumuganathan KEarle E. Nuclear DNA content of some important plant species. Plant molecular biology reporter 1991;9(3):208-218.

- Capela D, Marchetti M, Clérissi C, Perrier A, Guetta D, Gris C, Valls M, Jauneau A, Cruveiller S, Rocha EP. Recruitment of a lineage-specific virulence regulatory pathway promotes intracellular infection by a plant pathogen experimentally evolved into a legume symbiont. Molecular biology and evolution 2017;34(10):2503-2521.

- Daunay M-C. Eggplant. Vegetables II: Springer; 2008. p. 163-220.

- Gaitonde SRamesh R. Genetic characterization of Ralstonia solanacearum infecting eggplant Solanum melongena L. from Goa and Western region of India. 
- Genin S. Molecular traits controlling host range and adaptation to plants in Ralstonia solanacearum. New Phytologist 2010;187(4):920-928.

- Gopalakrishnan C, Singh T, Artal RB. Evaluation of eggplant accessions for resistance to bacterial wilt caused by Ralstonia solanacearum (EF Smith) Yabuuchi et al. Journal of Horticultural Science 2014;9(2):202-205.

- Hedges LLister C. Nutritional attributes of spinach, silver beet and eggplant. Crop Food Res Confidential Rep 2007;1928.

- Hirakawa H, Shirasawa K, Miyatake K, Nunome T, Negoro S, Ohyama A, Yamaguchi H, Sato S, Isobe S, Tabata S. Draft genome sequence of eggplant (Solanum melongena L.): the representative solanum species indigenous to the old world. DNA research 2014;21(6):649-660.

- Lebeau A, Daunay M-C, Frary A, Palloix A, Wang J-F, Dintinger J, Chiroleu F, Wicker E, Prior P. Bacterial wilt resistance in tomato, pepper, and eggplant: genetic resources respond to diverse strains in the Ralstonia solanacearum species complex. Phytopathology 2011;101(1):154-165.

- Liu H, Kang Y, Genin Sp, Schell MA, Denny TP. Twitching motility of Ralstonia solanacearum requires a type IV pilus system. Microbiology 2001;147(12):3215-3229.

- Monteiro F, Solé M, van Dijk I, Valls M. A chromosomal insertion toolbox for promoter probing, mutant complementation, and pathogenicity studies in Ralstonia solanacearum. Molecular plant-microbe interactions 2012;25(4):557-568.

- Nahar K, Matsumoto I, Taguchi F, Inagaki Y, Yamamoto M, Toyoda K, Shiraishi T, Ichinose Y, Mukaihara T. R alstonia solanacearum type III secretion system effector R ip36 induces a hypersensitive response in the nonhost wild eggplant $\mathrm{S}$ olanum torvum. Molecular plant pathology 2014;15(3):297-303.

- Persat A, Inclan YF, Engel JN, Stone HA, Gitai Z. Type IV pili mechanochemically regulate virulence factors in Pseudomonas aeruginosa. Proceedings of the National Academy of Sciences 2015:201502025.

- Ramesh R, Joshi A, Ghanekar M. Pseudomonads: major antagonistic endophytic bacteria to suppress bacterial wilt pathogen, Ralstonia solanacearum in the eggplant (Solanum melongena L.). World Journal of Microbiology and Biotechnology 2009;25(1):47-55.

- Ray SK, Kumar R, Peeters N, Boucher C, Genin S. rpoN1, but not rpoN2, is required for twitching motility, natural competence, growth on nitrate, and virulence of Ralstonia solanacearum. Frontiers in microbiology 2015;6:229.

- Salgon S, Jourda C, Sauvage C, Daunay M-C, Reynaud B, Wicker E, Dintinger J. Eggplant resistance to the Ralstonia solanacearum species complex involves both broadspectrum and strain-specific quantitative trait loci. Frontiers in plant science 2017;8:828.

- Shekhawat G, Singh R, Kishore V. Distribution of bacterial wilt and races and biotypes of the pathogen in India. JIPA, Journal of the Indian Potato Association 1978.

- Singh N, Phukan T, Sharma P, Kabyashree K, Barman A, Kumar R, Sonti RV, Genin S, Ray S. An innovative root inoculation method to study Ralstonia solanacearum pathogenicity in tomato seedlings. Phytopathology 2018;108(4):436-442.

- Siri MI, Sanabria A, Boucher C, Pianzzola MJ. New Type IV Pili-Related Genes Involved in Early Stages of Ralstonia solanacearum Potato Infection. Molecular PlantMicrobe Interactions 2014;27(7):712-724. 
- Smith EF. A bacterial disease of the tomato, eggplant and Irish potato. US Dept Agric Div Veg Phys Path Bull 1896;12:1-26.

- Syfert MM, Castañeda-Álvarez NP, Khoury CK, Särkinen T, Sosa CC, Achicanoy HA, Bernau V, Prohens J, Daunay MC, Knapp S. Crop wild relatives of the brinjal eggplant (Solanum melongena): poorly represented in genebanks and many species at risk of extinction. American journal of botany 2016;103(4):635-651.

- Wicker E, Grassart L, Coranson-Beaudu R, Mian D, Guilbaud C, Fegan M, Prior P. Ralstonia solanacearum strains from Martinique (French West Indies) exhibiting a new pathogenic potential. Applied and environmental microbiology 2007;73(21):6790-6801. 